\title{
AVALIAÇÃO DA PRESENÇA DE CUPINS NA ARBORIZAÇÃO DA REGIÃO CENTRAL DE PATO BRANCO-PR.
}

\author{
Ionete Hasse ${ }^{1}$; Tobias Juan Shinosaka ${ }^{2}$; Lenir Maristela Silva ${ }^{3}$
}

(recebido em 25.10.2007 e aceito pra publicação em 26.02.2008)

\begin{abstract}
RESUMO
No Brasil existem poucos dados referentes aos prejuízos causados por cupins na arborização urbana. Em Pato Branco-PR, bem como na maioria das cidades do Sudoeste do Paraná, a arborização urbana implantada na década de 1970 foi realizada sem nenhum planejamento e com a predominância da espécie exótica Ligustrum lucidum W.T. Aiton. O objetivo deste trabalho foi diagnosticar a presença de cupins na arborização central de Pato Branco-PR. Todos os espécimes arbóreos do bairro centro de Pato Branco-PR foram visualmente analisados e quando constatada a presença de cupins foram fotografados e catalogados em planilha. De agosto de 2006 a março de 2007 foram percorridas trinta e quatro ruas e analisadas 3.191 árvores. Os resultados dessa investigação apontam graves problemas com infestação de cupins nas três espécies arbóreas mais abundantes no bairro Centro de Pato Branco-PR. A espécie Ligustrum lucidum W.T. Aiton, mais abundante $(62,4 \%)$ na arborização urbana de Pato Branco-PR apresentou uma alta freqüência de infestação de cupins $28,67 \%$. A segunda espécie mais freqüente na arborização Lagerstroemia indica L. (11,4 \%) apresentou uma infestação de $8,72 \%$ e Bauhinia variegata L. $(3,8 \%)$ com uma infestação de 2,56\%. A existência de cupins indica um alto risco de queda do espécime, pois a presença dos mesmos ou orifícios visíveis no tronco comprometem as propriedades físicas e mecânicas diminuindo a resistência da árvore.
\end{abstract}

Palavras-chave: arborização urbana, Coptotermes spp e Ligustrum lucidum.

\footnotetext{
1 . Doutora em Produção Vegetal, UTFPR, Pato Branco/PR, hasse@utfpr.edu.br, Fone: (46) 32202511, Ramal: 2601, Fax (46) 3220-2500.

2 . Aluno do Ensino Médio, UTFPR Pato Branco/PR, bolsista PIBIC-JUNIOR/Fundação Araucária.

3 . Doutora em Produção Vegetal, UTFPR, Pato Branco/PR.
} 


\title{
EVALUATION OF PRESENCE OF TERMITES IN THE DOWNTOWN AREA TREE PLANTATION IN PATO BRANCO - PR.
}

\begin{abstract}
In Brazil, there aren't data referring to the damaged caused by termites in downtown tree plantation. In Pato Branco-PR, as well as in most of the cities of the Southwest region of Paraná, urban tree plantation implanted in the 1970's was held without any planning and with the predominance of the exotic species Ligustrum lucidum W.T. Aiton. The aim of this work was to diagnose the presence of termites in downtown tree plantation in Pato BrancoPr. All the arboreal specimens in the downtown area of Pato Branco were visually analyzed and when the presence of termites was evidenced the specimens were photographed and catalogued in a chart. From August, 2006 to March, 2007 thirty-four streets were investigated and 3.191 trees were analyzed. The results of this investigation point out severe problems with the infestation of termites in the most abundant arboreal species in the downtown are of Pato Branco. The species Ligustrum lucidum W.T. Aiton, most abundant $(62,4 \%)$ in urban tree plantation of Pato Branco has shown a high frequency of infestation of termites $28,67 \%$. The second most frequent species in tree plantation Lagerstroemia indica L. $(11,4 \%)$ has shown an infestation of $8,72 \%$ and Bauhinia variegate L. $(3,8 \%)$ with an infestation of $2,56 \%$. The existence of termites indicates a high risk of fall of the specimen since their presence or visible orifices in the tree trunk compromise the physical and mechanical properties reducing the resistence of the tree.
\end{abstract}

Key-words: urban tree plantation; Coptotermes spp and Ligustrum lucidum. 


\section{INTRODUÇÃO}

Os cupins são insetos importantes na ciclagem da matéria orgânica e na formação do solo. Contudo ao perderem seu habitat frente ao avanço das cidades se proliferam devido à eliminação dos competidores e dos predadores naturais pelo homem (WALLER e LA FAGE 1986). Segundo Constantino (2002) de 2.861 espécies de térmitas existentes no mundo, cerca de 70 ou 80 espécies são consideradas pragas.

No Brasil existem poucos estudos sobre a infestação de cupins na arborização urbana. Segundo Fontes (1995) os cupins são a causa de grandes prejuízos para a arborização urbana, sendo que as árvores constituem um abrigo para estes insetos.

Edificações infestadas por Coptotermes gestroi Wasmann são encontradas nos grandes centros urbanos como São Paulo e Rio de Janeiro, espécie de cupim originária do sudeste da Ásia, que se adaptou muito bem às condições urbanas (TIEPPO et al., 2007). Os mesmos autores citam que existem relatos da infestação de cupins em árvores na cidade de São Paulo, como por exemplo, em Sibipiruna, Jacarandá-mimoso, Quaresmeira, Acácia, Ipê e Paineira.

Segundo Amaral (2002), o problema de cupins xilófagos nas edificações e prédios históricos, na cidade de São Paulo, Brasil, não traz conseqüências apenas para estas estruturas, mas comprometem a sanidade biológica das árvores que por serem atacadas tornam-se mais sujeitas as quedas, podendo colocar em risco à integridade das pessoas, animais, veículos e edificações.

Brazolin (2006) cita que no Brasil, os cupins xilófagos, aqueles que atacam a madeira, do grupo dos "cupins-subterrâneos" e da espécie Coptotermes gestroi (antigo C. havilandi), são responsáveis por grandes prejuízos à arborização urbana e as árvores constituem um abrigo para estes insetos, não sendo rara a constatação de ninhos destes cupins no interior de troncos ocos de árvores.

Segundo Amaral (2002) os indícios da ocorrência de cupins são vestígios ou túneis de cupins subterrâneos na superfície do tronco, interna ou externa à casca. Contudo os cupins subterrâneos da espécie Coptotermes havilandi podem estar presentes nas árvores apenas internamente e não serem observados pela análise externa. O mesmo autor realizou estudo de prospecção interna do tronco por meio de uma avaliação não destrutiva para estimar a intensidade da infestação interna da árvore.

Infelizmente, em Pato Branco, bem como na maioria das cidades do Sudoeste do Paraná, a arborização urbana implantada na década de 1970 foi realizada sem nenhum planejamento e com espécies exóticas com alto potencial de invasoras. Segundo Silva et al (2007), em Pato Branco houve uma implantação generalizada de uma única espécie exótica na arborização urbana: o ligustro (Ligustrum lucidum W.T. Aiton). Com o decorrer dos anos 
e o conseqüente crescimento das espécies, começaram a ocorrer conflitos das árvores com a infraestrutura urbana, principalmente, com a rede elétrica que apresenta o padrão tradicional de fiação. Diante dos problemas, sazonalmente houve medidas emergenciais, como arranquio e podas drásticas de inúmeros indivíduos arbóreos.

As podas mal executadas ou drásticas propiciam a entrada de cupins, uma vez que a injúria expõe o lenho e permite a instalação desses organismos (BRAZOLIN, 2006). Na África Coptotermes spp. são frequentemente encontrados nesta situação (HARRIS, 1971).

A existência de cupins indica um alto risco da queda do espécime, pois a presença dos mesmos ou orifícios visíveis no tronco comprometem as propriedades físicas e mecânicas diminuindo sua resistência. $O$ objetivo deste trabalho foi diagnosticar a presença de cupins na arborização central de Pato Branco-PR.

\section{MATERIAL E MÉTODOS}

A área inventariada localiza-se em Pato Branco, Paraná, no bairro Centro. Pato Branco situa-se na região sudoeste do Paraná, no terceiro planalto paranaense. Segundo a classificação de Köppen o clima é do tipo Cfa - Clima subtropical Úmido Mesotérmico, destacando-se o solo do tipo nitossolo (EMBRAPA, 1999). Pato Branco encontra-se a 760 m. de altitude, com Latitude de 26 13' 46" - Sul e Longitude de 52 40' 14" - W-GR. A população total corresponde a 62.167 habitantes, sendo que destes 56.739 , correspondem a população urbana (IBGE, 2000). A área urbana apresenta 71,23 Km². Caracteriza-se como residencial e comercial e não possui cadastro histórico da sua arborização.

Para localização das vias públicas, bem como a obtenção de suas extensões, foi utilizado um mapa (Figura1 ) elaborado pelo IPPUPB (Instituto de Pesquisa e Planejamento Urbano de Pato Branco). 


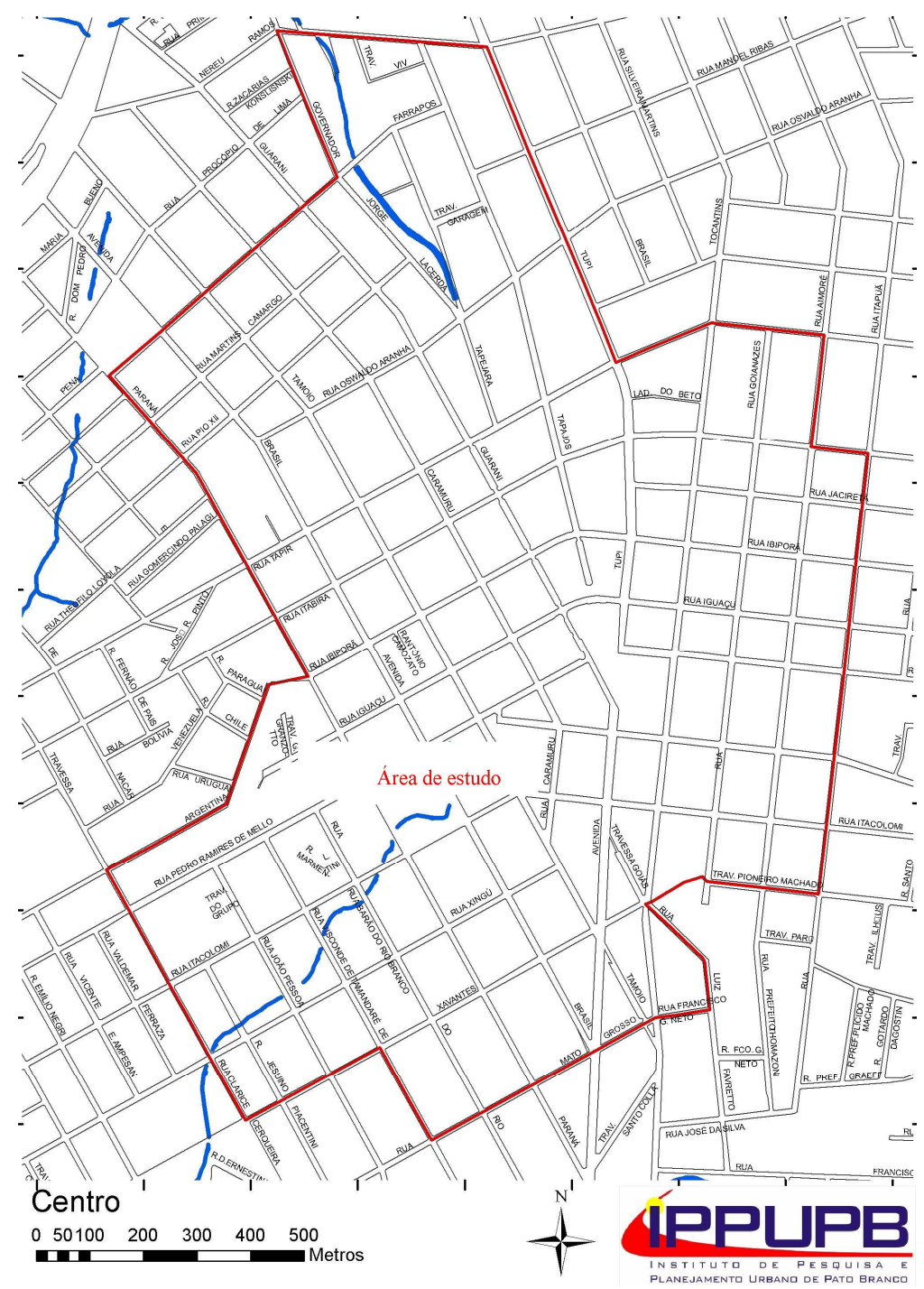

FIGURA 1-Planta planialtimétrica do centro de Pato Branco na escala 1:10.000, elaborada pelo IPPUPB (Instituto de Pesquisa e Planejamento Urbano de Pato Branco-PR) 2005.

A vegetação da região na qual se localiza a área urbana foi originariamente coberta por Floresta Ombrófila Mista Montana. Esta formação ocupa as regiões planálticas do Paraná, apresentando faixa de ocorrência altitudinal entre 400 e 1.000 metros. O dossel contínuo varia em torno de 20 metros de altura, mas as araucárias atingem até 25 metros. A Floresta Ombrófila Mista também é conhecida por "Floresta de Pinheiros", "Pinheirais", "Zona dos Pinhais", "Matas de Araucária" e "Florestas com Araucária" (IBGE, 1992).

Esse trabalho faz parte de um projeto amplo que inventariou vários aspectos inerentes à arborização da cidade de Pato Branco-PR. Todos os espécimes arbóreos anteriormente identificados por Silva et al. (2007a) e Silva et al. (2007b) foram avaliadas quanto à presença e ausência de cavidades e pó de cupim no tronco e nos ramos das árvores. As árvores que apresentaram presença de cupins foram fotografadas e catalogadas 
em planilha. De agosto de 2006 a março de 2007 foram percorridas trinta e quatro ruas (Figura1) e analisadas 3.191 árvores.

\section{RESULTADOS E DISCUSSÃO}

As 3.191 árvores presentes na arborização central de Pato Branco-PR são de 47 espécies diferentes, sendo que três espécies apresentaram infestação por cupins (Tabela 01).

A espécie exótica Ligustrum lucidum W.T. Aiton de maior predominância na arborização Central de Pato Branco-PR, e implantada na década de 1970, foi a que apresentou maior freqüência de infestação por cupins (Tabela 01).

As espécies Lagerstroemia indicam L. e Bauhinia variegata L. (Tabela 01) implantadas na arborização central de Pato Branco-PR após o Ligustrum lucidum W.T. Aiton (Figura $2 \mathrm{~A}$ ), demonstraram ser suscetíveis ao ataque de cupins, principalmente a Lagerstroemia indica L. (Figura 2 B), espécie exótica muito observada na arborização das cidades do sudoeste do Paraná.

TABELA 01 - Freqüência das espécies arbóreas no bairro Centro de Pato Branco (PR) e porcentagem de infestação por cupins.

\begin{tabular}{ccccc}
\hline Espécie & $\begin{array}{c}\text { Número de } \\
\text { espécimes }\end{array}$ & $\begin{array}{c}\text { Freqüência } \\
(\%)\end{array}$ & $\begin{array}{c}\text { Presença de } \\
\text { cupins } \\
(\%)\end{array}$ & $\begin{array}{c}\text { Freqüência de } \\
\text { ataque de } \\
\text { cupins na } \\
\text { espécie }\end{array}$ \\
\hline Ligustrum lucidum W.T. Aiton & 1.992 & 62,4 & 28,67 & 45,94 \\
Lagerstroemia indica L. & 363 & 11,4 & 8,72 & 76,49 \\
Schinus molle L & 200 & 6,3 & 0 & 0 \\
Bauhinia variegata L. & 121 & 3,8 & 2,56 & 67,36 \\
Outras & 515 & 16,1 & 0 & 0 \\
\hline TOTAL & 3.191 & 100 & 39,95 &
\end{tabular}

Obs: A tabela com a lista de todas as espécies foi publicada por Silva et al. (2007a). 


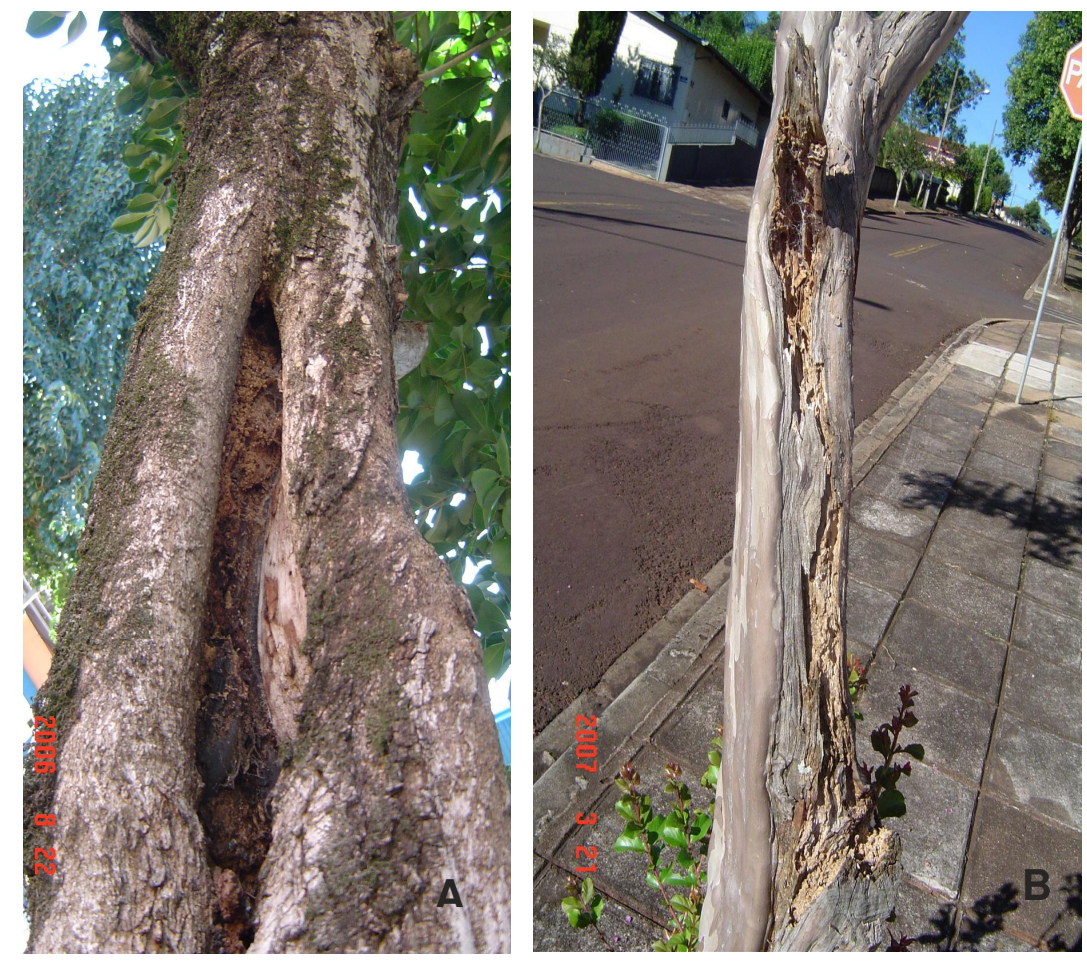

FIGURA 2 - Presença de cupins em troncos de Ligustrum lucidum W.T. Aiton (A) e em Lagerstroemia indica L (B) na arborização central de Pato Branco-PR/2006.

Brazolin (2006) constatou que o Ligustrum lucidum (alfeneiro) estava entre as espécies de maior predominância nos passeios públicos de São Paulo e foi considerada como uma das três espécies mais susceptíveis ao ataque de organismos xilófagos. Segundo esse autor o estado de sanidade biológica da árvore, alterada principalmente pela ação de cupins-subterrâneos (Coptotermes gestroi) ou por fungos apodrecedores, está relacionado à ruptura das árvores, quando esta é submetida a esforços acima de seu limite de resistência.

A infestação por cupins nas espécies exóticas Ligustrum lucidum W.T. Aiton e Lagerstroemia indicam $L$ (Tabela 01) pode estar relacionada à predominância das espécies na arborização, como também pelo manejo inadequado, como, por exemplo, as podas drásticas observadas principalmente na espécie Ligustrum lucidum W.T. Aiton. Segundo Becker (1975), as podas em árvores ornamentais das ruas ou árvores frutíferas que se encontram plantadas em áreas infestadas por cupins, facilitam o ataque de fungos e cupins, que penetram através dos cortes, acionando um ataque intenso.

Atualmente a espécie Ligustrum lucidum W.T. Aiton, não é recomendada para a arborização urbana devido ao cultivo excessivo e ao potencial dispersor (BACKES e IRGANG, 2004). Segundo Instituto Hórus (2005), no Paraná essa espécie é invasora da Floresta Ombrófila mista, bioma da região de Pato Branco-PR. O Ligustrum lucidum W.T é 
citado na lista oficial de espécies exóticas invasoras no Estado do Paraná (IAP, 2007) que estabelece normas de controle e erradicação para evitar a contaminação biológica.

A espécie nativa Schinus molle L (Tabela 01) não apresentou infestação por cupins e pode ser recomendada para substituir o Ligustrum lucidum W.T. Aiton. Outras espécies nativas do Brasil que podem substituir o ligustro e que apresentam de pequeno a médio portes (Silva et al. 2007a), com baixa freqüência de indivíduos na arborização central de Pato Branco-PR e que não apresentaram infestação por cupins (Tabela 01) podem ser, por exemplo: Eugenia uniflora L., Lafoensia pacari St. Hil., Psidium catleianum Sabine, Rollinia rugulosa Schlecht, Tabebuia heptaphylla (Vell.) Tol.

\section{CONCLUSÕES}

- Podas freqüentes e drásticas observadas na espécie Ligustrum lucidum W.T. Aiton favoreceram a entrada de cupins.

- Novos estudos visando à coleta e classificação dos cupins na área urbana de Pato Branco-PR, devem ser executados nas próximas pesquisas.

- Recomenda-se substituição gradativa das espécies infestadas com risco de queda pela biodeterioração causada pelos cupins.

\section{AGRADECIMENTOS}

Ao Programa Institucional de Iniciação Científica do CNPq (PIBIC/Fundação Araucária) pelo financiamento de uma bolsa ao aluno PIBIC-Junior.

\section{REFERÊNCIAS BIBLIOGRÁFICAS}

AMARAL, R.D.A.M. Diagnóstico da ocorrência de cupins xilófagos em árvores urbanas no bairro de Higienópolis, na cidade de São Paulo. Piracicaba: 88 f. Dissertação (Mestrado em Recursos Florestais) Escola Superior de Agricultura "Luís de Queiroz", Universidade de São Paulo, 2002.

BECKER, G. On termites in Central and South America. Revista Floresta, v.9, n.2, p.71-75, 1978.

BACKES, P.; IRGANG, B. Árvores cultivadas no sul do Brasil: Guia de identificação e interesse paisagístico das principais espécies exóticas. Santa Cruz do Sul: Instituto Souza Cruz, 2004. 204 p. 
BRAZOLIN. S., Biodeterioração de árvores urbanas e análise de risco de queda. In: minicursos. X Congresso Brasileiro de Arborização Urbana Maringá-PR. CD Congresso, Novembro de 2006.

CONSTANTINO, R. The pest termites of South America: taxonomy, distribution and status. Journal of Applied Entomology. 126, p. 355-365, 2002.

EMPRESA BRASILEIRA DE PESQUISA AGROPECUÁRIA -. Centro Nacional de Pesquisa de Solos. Sistema Brasileiro de Classificação de Solos. Brasília, 1999. 412 p.

FONTES, L.R. Termites (Isoptera) que causam infestação no Brasil. In: BERTI FILHO, E. (Ed.); FONTES, L. R. (Ed.). Alguns aspectos atuais da biologia e controle de cupins. Piracicaba. FEALQ, 1995. p.163-164.

HARRIS, W.V. Termites: their recognition and control. England: Longman Group, 1971. $186 p$.

IAP - INSTITUTO AMBIENTAL DO PARANÁ. PORTARIA IAP N 095. 2007. Disponível em $<$ http://www.iap.pr.gov.br/arquivos/File/iap/port 95 07.pdf> Acesso em 20//02/2008.

BGE - INSTITUTO BRASILEIRO DE GEOGRAFIA E ESTATÍSTICA. Manual técnico da vegetação brasileira. Rio de Janeiro: IBGE, 1992. 92 p.

IBGE - INSTITUTO BRASILEIRO DE GEOGRAFIA E ESTATístICA. Atlas do senso demográfico. Rio de Janeiro: IBGE, 2000. 127p.

INSTITUTO HÓRUS DE DESENVOLVIMENTO E CONSERVAÇÃO AMBIENTAL /THE NATURE CONSERVANCY. Ligustrum Iucidum. 2005. Disponível em $<$ http://www.institutohorus.org.br/download/fichas/Ligustrum_lucidum.htm> Acesso em 12/02/2007.

SILVA M.L.; HASSE, I.; MOCCELLIN, R.; ZBORALSKI R.A. Arborização de vias públicas e a utilização de espécies exóticas: o caso do bairro centro de Pato Branco-PR. Scientia agrária, v.8, n. 2, 2007a.

SILVA, L. M. ; MOCCELLIN, Renata ; WEISHEIMER, Daiana IIma ; ZBORALSKI, Adriane Rodrigues ; FONSECA, Lidiane ; RODIGHIERO, Denielly Arruda . Inventário e sugestões 
para arborização em via pública de Pato Branco/PR. Revista da Sociedade Brasileira de Arborização Urbana, v. 2, p. 101-108, 2007b.

TIEPPO, F.M.M.; TREVISAN, H.; CARVALHO A.G. Resistência da madeira de quatro essências utilizadas na arborização urbana a Coptotermes Gestroi (WASMANN, 1896) Revista da Sociedade Brasileira de Arborização Urbana, v.2, n.1, 2007.

WALLER, D.A.; LA FAGE, J.P. Nutritional ecology of termites. In: SLANSKY JR., F.;RODRIGUEZ, J. C. Nutritional ecology of insects, mites, spiders and related invertebrates. New York: Wiley-Interscience Publication, 1986. Cap.16, p.487-532. 